\title{
Translational invariance of the Einstein-Cartan action in any dimension
}

\author{
N. Kiriushcheva \\ Department of Applied Mathematics, \\ University of Western Ontario, London, Canadd* \\ S.V. Kuzmin \\ Faculty of Arts and Social Science, \\ Huron University College and Department of Applied Mathematics, \\ University of Western Ontario, London, Canadd
}

(Dated: November 7, 2018)

\begin{abstract}
We demonstrate that from the first order formulation of the Einstein-Cartan action it is possible to derive the basic differential identity that leads to translational invariance of the action in the tangent space. The transformations of fields is written explicitly for both the first and second order formulations and the group properties of transformations are studied. This, combined with the preliminary results from the Hamiltonian formulation (arXiv:0907.1553 [gr-qc]), allows us to conclude that without any modification, the Einstein-Cartan action in any dimension higher than two possesses not only rotational invariance but also a form of translational invariance in the tangent space. We argue that not only a complete Hamiltonian analysis can unambiguously give an answer to the question of what a gauge symmetry is, but also the pure Lagrangian methods allow us to find the same gauge symmetry from the basic differential identities.
\end{abstract}

*Electronic address: nkiriush@uwo.ca

${ }^{\dagger}$ Electronic address: skuzmin@uwo.ca 


\section{INTRODUCTION}

From the Hamiltonian analysis of the first order form of the Einstein-Cartan (EC) action without coupling to matter and using the N-beins and connections as independent variables, it is clear that the only gauge invariance that follows from the first class constraints, in addition to the rotational ("Lorentz") invariance in the tangent space, is some form of translational invariance also in the tangent space (for some preliminary results using the Hamiltonian analysis see [1]). In all dimensions higher than two this invariance must possess a parameter with an internal index that corresponds to either the translational part of the Poincaré symmetry (as in the three dimensional case [2]) or a Poincaré symmetry with a "more general group structure that the original Poincaré group" [3]. In a flat spacetime limit this "more general group structure" is equivalent to the Poincaré group, providing a natural generalization of Poincaré symmetry to curved spacetime [4]. In supergravity a translational invariance in the tangent space was used for the first time by Breitenlohner [5] to avoid "on-shell" closure of the commutator algebra. The paper which is most closely related to our analysis (to both Hamiltonian [1, 2] and Lagrangian analysis) is one by Teitelboim [6], where translational and rotational invariances in the tangent space were discussed and the structure of the algebra of constraints for both gravity and supergravity, as well as the resultant transformations of fields, were predicted using very general observations. The explicit form of either the constraints or the structure functions were not given in [6], but our Hamiltonian analysis of the EC action leads exactly to such a structure.

However a different opinion commonly appears in the literature about the ordinary EC action (without considering any generalization and supersymmetrizations). For example, in [7] it is stated that the EC action "is invariant under Lorentz rotations and diffeomorphisms, but not under translations [italic of M.B.]", and that only in three dimensions is the EC action a gauge theory with both rotational and translational invariances. According to [8], “... a closer analysis shows that only Lorentz rotations are symmetries of the action: the translational part of the Poincaré group does not leave the action invariant" and "...gravity is not a gauge theory..."

The statements that there is no translational invariance in dimensions higher than three have appeared in the literature without proof, but quite often the reference on Witten's work [9] is used. However, Witten did not draw this conclusion, only stating: "we cannot hope that 
four-dimensional gravity would be a gauge theory in this sense" (in the sense that it would be similar to the Chern-Simons action). We do not see how this statement was generalized to saying that EC gravity is not a gauge theory in dimensions higher than three [8] or that it is a theory with diffeomorphism as a gauge symmetry [8, 10 12] (with transformations in the external space), in place of translational invariance in the internal space, which is a part of Poincaré or generalized Poincaré symmetry. ${ }^{1}$ In many papers the authors switch without justification from the translational invariance in the tangent space (which naturally arises from the constraint structure in the first steps of the Hamiltonian formulation [1, 2], as well as from the basic differential identities of the Lagrangian formulation (see next Section)) to diffeomorphism invariance (translation in the external space) in an attempt to try to obtain the "expected" result, for further "convenience" (see, e.g., [8, 10, 12 14]; this is even done for the EC action in three dimensions [9, 15]). The EC action is manifestly diffeomorphism invariant but this is not necessary a gauge invariance if by "gauge invariance" we mean a transformation generated by the first class constraints of the theory. It has been stated that there exist various ways to define the constraints of tetrad gravity which lead to different gauge transformations [16]. However, at the Hamiltonian level, such changes are possible only if one makes non-canonical transformations of variables. This erroneously assumes that a unique characteristic of a theory, namely the gauge invariance which follows from the first class constraints of the theory, can be chosen according to one's preference.

All symmetries of the EC action are encoded in it (as in any field theory) and should be found without any a priori knowledge of what symmetry we expect. One of many symmetries of EC action is translational invariance (contrary to what is stated in some references) and we will show that this symmetry follows from the Lagrangian in the most natural way, just as the rotational symmetry can be derived from the Lagrangian, and there is no need to modify the action to have such an invariance. The question whether translation is a gauge symmetry which follows from the first class constraints of the EC action or it "is not a gauge theory" [8] can be answered by using Hamiltonian methods.

In the Hamiltonian approach to constrained (singular) systems [17] there is the welldefined criterion for a theory being a gauge theory: if first class constraints are present

\footnotetext{
${ }^{1}$ In some papers Lorentz symmetry plus diffeomorphism are erroneously called the Poincaré gauge symmetry (see, for example, [10, 12]).
} 
then the theory possesses a gauge symmetry that can be found uniquely from the Poisson brackets $(\mathrm{PBs})$ algebra of these first class constraints. To find this gauge symmetry and the associated transformations of the fields, second class constraints have to be eliminated (the Hamiltonian reduction), and by applying some standard methods (e.g. the oldest one due to Castellani [18]), gauge transformations of the fields can be obtained. Of course, the Hamiltonian procedure destroys manifest covariance, especially after elimination of the second class constraints (which are always present in first order formulations), and if the gauge transformations are covariant then restoring their covariant form requires labourious calculations using solutions of second class constraints. (For some examples see [19], [2], and [1].)

In contrast, if one is not interested in obtaining the reduced Hamiltonian by elimination of non-physical degrees of freedom, the explicit form of the constraints, the algebra of PBs, etc., and is only interested in finding all symmetries of an action without determining which of the symmetries is a gauge symmetry generated by the first class constraints and which is not, then Lagrangian methods are preferable as they retain manifest covariance making calculations much simpler and more transparent. In some works (e.g. see a recent article of Samanta [20] and references therein), there are examples of such "Lagrangian" approaches. However, in [20], covariance is needlessly broken and the approach presented is just a modification of the Hamiltonian method of Castellani [18]. Such a modification is unnecessary, especially because in applications of this "Lagrangian" method differential identities (DIs) are taken for granted [20] and they are always in covariant form. If DIs are known, the corresponding transformations of the fields are simply obtained. A method of finding a general DI does not exist in these approaches; artificially destroying covariance in such "Lagrangian" methods makes this task impossible without a priori knowledge of the symmetries of the theory. Indeed, if one uses known symmetries of the theory in conjunction with the corresponding DIs, one can obtain the associated Noether current in the theory [21].

In model building many approaches are based on postulating particular symmetries and constructing actions that support them. Our goal is different: using the EC action as an example, we demonstrate that there is an algorithm for building differential identities that allows us to find all symmetries of an action without a priori knowledge of what symmetries it has (not to build an action that should possess some symmetries). We explicitly demonstrate 
that statements that translational invariance in dimensions higher than three is absent are groundless by deriving such transformations for the EC action. The translational invariance that we find has been observed in [3, 4, 22].

In this paper, using the purely Lagrangian approach (Section II) we find the basic DIs which directly lead to both rotational and translational invariances in the tangent space for the EC action in all dimensions higher than three. In Section III the group structure of some invariances and their different combinations are discussed. Using the equivalence of the Lagrangian and Hamiltonian methods, in our conclusion we address the question "what is a gauge symmetry and what is not?" [23]. We argue that pure Lagrangian methods also can be used to unambiguously single out the same symmetry that follows from Hamiltonian methods (i.e. originates with the first class constraints). This is what we refer to as a gauge symmetry.

\section{TRANSLATIONAL INVARIANCE OF THE EINSTEIN-CARTAN ACTION}

The EC action in its first order form where N-bein fields $e_{\mu(\alpha)}$ and connections $\omega_{\mu(\alpha \beta)}=$ $\omega_{\mu(\beta \alpha)}$ are independent variables ${ }^{2}$ is (found, e.g., in [24, 25])

$$
S(e, \omega)=\int d^{D} x L(e, \omega)
$$

with the Lagrange density

$L(e, \omega)=-e A^{\mu(\alpha) \nu(\beta)}\left(\omega_{\nu(\alpha \beta), \mu}+\omega_{\mu(\alpha \gamma)} \omega_{\nu}^{(\gamma}{ }_{\beta}\right)=-e e^{\mu(\alpha)} e^{\nu(\beta)} R_{\mu \nu(\alpha \beta)}=-\frac{1}{2} e A^{\mu(\alpha) \nu(\beta)} R_{\mu \nu(\alpha \beta)}$

where $e=\operatorname{det}\left(e_{\gamma(\rho)}\right), D$ is the dimension of spacetime and

$$
A^{\mu(\alpha) \nu(\beta)}=e^{\mu(\alpha)} e^{\nu(\beta)}-e^{\mu(\beta)} e^{\nu(\alpha)}=-A^{\mu(\beta) \nu(\alpha)}=-A^{\nu(\alpha) \mu(\beta)} .
$$

The properties of $A^{\mu(\alpha) \nu(\beta)}$ and other similar functions which considerably simplify calculations are presented in Appendix A.

\footnotetext{
${ }^{2}$ Usually variables $e_{\gamma(\rho)}$ and $\omega_{\nu(\alpha \beta)}$ are called tetrads and spin connection, but such names are specialized for $D=4$. As we consider the Lagrangian formulation in any dimension $(D>2)$, we will call $e_{\gamma(\rho)}$ and $\omega_{\nu(\alpha \beta)}$ N-beins and connections, respectively.
} 
We also use the Ricci tensor, antisymmetric in $\alpha \beta$ and $\mu \nu$,

$$
R_{\mu \nu(\alpha \beta)}=\omega_{\nu(\alpha \beta), \mu}-\omega_{\mu(\alpha \beta), \nu}+\omega_{\mu(\alpha \gamma)} \omega_{\nu \beta}^{(\gamma}-\omega_{\nu(\alpha \gamma)} \omega_{\mu}^{(\gamma}{ }_{\beta}=-R_{\nu \mu(\alpha \beta)}=-R_{\mu \nu(\beta \alpha)} .
$$

Indices in brackets (..) denote the internal ("Lorentz") indices, and indices without brackets are external or "world" indices. Internal and external indices are raised and lowered by the Minkowski tensor $\eta_{(\alpha)(\beta)}=(-,+,+, \ldots)$ and the metric tensor $g_{\mu \nu}=e_{\mu(\alpha)} e_{\nu}^{(\alpha)}$, respectively. We assume that the inverse $e^{\mu(\alpha)}$ exists and $e^{\mu(\alpha)} e_{\gamma(\alpha)}=\delta_{\gamma}^{\mu}, e^{\mu(\alpha)} e_{\mu(\beta)}=\delta_{(\beta)}^{(\alpha)}$. Note that neither the contravariant N-bein $e^{\mu(\alpha)}$ nor the metric tensor $g_{\mu \nu}$ are independent variables.

Variation of the action (11) with respect to the independent fields is given by

$$
\delta S=\int d^{D} x\left(E^{\mu(\alpha)} \delta e_{\mu(\alpha)}+E^{\mu(\alpha \beta)} \delta \omega_{\mu(\alpha \beta)}\right)=0
$$

where the Euler derivatives (EDs) are

$$
\begin{aligned}
& E^{\tau(\rho)}=\frac{\delta L}{\delta e_{\tau(\rho)}}=-e B^{\tau(\rho) \mu(\alpha) \nu(\beta)}\left(\omega_{\nu(\alpha \beta), \mu}+\omega_{\mu(\alpha \gamma)} \omega_{\nu}{ }^{(\gamma} \beta\right) \\
& \left.E^{\tau(\rho \sigma)}=\frac{\delta L}{\delta \omega_{\tau(\rho \sigma)}}=e B^{\nu(\alpha) \mu(\rho) \tau(\sigma)} e_{\nu(\alpha), \mu}-e A^{\tau(\rho) \nu(\beta)} \omega_{\nu}{ }^{(\sigma)}+e A^{\tau(\sigma) \nu(\beta)} \omega_{\nu}{ }^{(\rho} \beta\right)^{\circ} .
\end{aligned}
$$

Note that the EDs are the result of variation of an action with respect to the independent fields. In this $e=\operatorname{det}\left(e_{\gamma(\rho)}\right)$ is also the part of the variation, and thus the EDs are different from equations of motion (see, e.g. [20]) where $e$ can be omitted, and other rearrangements can be performed such as contraction with fields, taking different combinations of equations of motion, etc.

Systematic studies of symmetries of any action can be performed by constructing differential identities which are linear combinations of EDs. DIs identically equal zero without using solutions of the corresponding equations of motion (they are valid "off-shell"). In general, coefficients of the EDs in such linear combinations are functions of the fields and/or their derivatives. Such differential identities (if found) immediately lead to an invariance of the action under the corresponding transformations of fields. For example, let us say, we construct an identity which has one external index $I^{\mu}=0$. Multiplying it by a parameter $\xi_{\mu}$ to form a scalar, we have

$$
\int d^{D} x I^{\mu} \xi_{\mu}=0
$$


and so, both equations (44) and (7) are equivalent. Because the DIs are constructed to be linear in the EDs, by performing integration(s) by parts and/or redefining dummy indices in (17) we can easily obtain

$$
\int d^{D} x[\underbrace{(\ldots)}_{\delta e_{\mu(\alpha)}} E^{\mu(\alpha)}+\underbrace{(\ldots)}_{\delta \omega_{\mu(\alpha \beta)}(\ldots)} E^{\mu(\alpha \beta)}]=\int d^{D} x\left(E^{\mu(\alpha)} \delta e_{\mu(\alpha)}+E^{\mu(\alpha \beta)} \delta \omega_{\mu(\alpha \beta)}\right)
$$

and we can just read off the transformation of the fields that correspond to a particular DI.

Construction of a DI is an iterative procedure and we will illustrate it by first finding DI that generates a rotational ("Lorentz") invariance which is well-known for N-bein gravity. Such a DI arises quite naturally, because the simplest DI that one can think of is to start from the derivative of the ED (6) . Let us consider $E_{\tau}^{\tau(\rho \sigma)}$ :

$$
\begin{aligned}
& E_{, \tau}^{\tau(\rho \sigma)}=-e B^{\mu(\alpha) \tau(\rho) \nu(\beta)} e_{\mu(\alpha), \tau} \omega_{\nu}^{(\sigma}{ }_{\beta}+e B^{\mu(\alpha) \tau(\sigma) \nu(\beta)} e_{\mu(\alpha), \tau} \omega_{\nu}{ }^{(\rho)} \\
& \left.-e A^{\tau(\rho) \nu(\beta)} \omega_{\nu}{ }^{(\sigma), \tau}+e A^{\tau(\sigma) \nu(\beta)} \omega_{\nu}^{(\rho)} \beta\right), \tau .
\end{aligned}
$$

(The first two terms in $E_{, \tau}^{\tau(\rho \sigma)}$ are $e C^{\lambda(\gamma) \nu(\alpha) \mu(\rho) \tau(\sigma)} e_{\lambda(\gamma), \tau} e_{\nu(\alpha), \mu}+e B^{\nu(\alpha) \mu(\rho) \tau(\sigma)} e_{\nu(\alpha), \mu \tau}$ (using (A10) for $\left.\left(e B^{\nu(\alpha) \mu(\rho) \tau(\sigma)}\right),{ }_{\tau}\right)$, but both of them vanish because of antisymmetry of $C$ and $B$ (see Appendix A).)

We start the iterative procedure by trying to express all terms with derivatives appearing in (9) in terms of EDs by comparison with the corresponding contributions in (5) and (6). Two first terms of (9) are exactly the first term in (6) contracted with a connection. The last two terms are not exactly equal to any of the EDs, but because of the presence of derivatives of connections, they can be related only to (5). It is obvious that trying to relate them to (5) we have to perform a contraction of $B$ with the N-bein field to obtain an expression similar to (A11). Because $E^{\tau(\rho)}$ has only one free external index $\tau$, then it must be a contraction of this index and this fixes the choice of the indices in the expansion of $B$ needed to simplify this calculation. Contraction of (5) with $e_{\tau}^{(\sigma)}$ gives

$$
\left.e_{\tau}^{(\sigma)} E^{\tau(\rho)}=-e e_{\tau}^{(\sigma)} B^{\tau(\rho) \mu(\alpha) \nu(\beta)} \omega_{\nu(\alpha \beta), \mu}-e e_{\tau}^{(\sigma)} B^{\tau(\rho) \mu(\alpha) \nu(\beta)} \omega_{\mu(\alpha \gamma)} \omega_{\nu}{ }^{(\gamma} \beta\right) .
$$

Using (A11) for the first term of the right-hand side of (10) and solving it for $e A^{\mu(\rho) \nu(\beta)} \omega_{\nu}^{(\sigma}{ }_{\beta), \mu}$ we obtain 


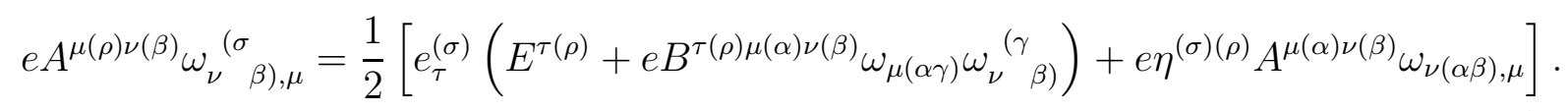

Substituting the expression $e A^{\mu(\rho) \nu(\beta)} \omega_{\nu}{ }^{(\sigma}{ }_{\beta, \mu}$ from (11) into the second line of (9) and using the identity

$$
e B^{\mu(\alpha) \tau(\rho) \nu(\beta)} e_{\mu(\alpha), \tau}=E^{\nu(\rho \beta)}+e A^{\nu(\rho) \tau(\lambda)} \omega_{\tau}^{(\beta)}-e A^{\nu(\beta) \tau(\lambda)} \omega_{\tau}^{(\rho)}
$$

in the first line of (9), gives us

$$
\left.E_{, \tau}^{\tau(\rho \sigma)}=-E^{\nu(\rho \beta)} \omega_{\nu}^{(\sigma} \beta\right)+E^{\nu(\sigma \beta)} \omega_{\nu}^{(\rho)}-\frac{1}{2} e_{\tau}^{(\sigma)} E^{\tau(\rho)}+\frac{1}{2} e_{\tau}^{(\rho)} E^{\tau(\sigma)}+W^{(\rho \sigma)}
$$

where, as a result of the first iteration, all terms with derivatives are expressed in terms of EDs. The remaining terms, all of them without derivatives, are collected in $W^{(\rho \sigma)}$ :

$$
\begin{aligned}
W^{(\rho \sigma)}=- & \left.\left.\left(e A^{\nu(\rho) \tau(\lambda)} \omega_{\tau}^{(\beta} \lambda\right)-e A^{\nu(\beta) \tau(\lambda)} \omega_{\tau}^{(\rho)}\right) \omega_{\nu}^{(\sigma)}+\left(e A^{\nu(\sigma) \tau(\lambda)} \omega_{\tau}^{(\beta}{ }^{(\beta)}-e A^{\nu(\beta) \tau(\lambda)} \omega_{\tau}^{(\sigma)}\right) \omega_{\nu}{ }^{(\rho} \beta\right) \\
& \left.-\frac{1}{2} e_{\tau}^{(\sigma)}\left[e B^{\tau(\rho) \mu(\alpha) \nu(\beta)} \omega_{\mu(\alpha \gamma)} \omega_{\nu}^{(\gamma}{ }^{(\rho)}\right]+\frac{1}{2} e_{\tau}^{(\rho)}\left[e B^{\tau(\sigma) \mu(\alpha) \nu(\beta)} \omega_{\mu(\alpha \gamma)} \omega_{\nu}{ }^{(\gamma} \beta\right)\right]
\end{aligned}
$$

After performing an expansion of $B$ and contracting with $e_{\tau}^{(\sigma)}$ using (A11), all terms have the same structure: $A \omega \omega$. A relabelling the dummy indices leads to a complete cancellation, so that $W^{(\rho \sigma)}=0$. The iterative procedure now stops and the rotational DI is

$$
I^{(\rho \sigma)}=E_{, \nu}^{\nu(\rho \sigma)}+E^{\nu(\rho \beta)} \omega_{\nu}^{(\sigma}{ }_{\beta}-E^{\nu(\sigma \beta)} \omega_{\nu \beta}^{(\rho}{ }^{(\rho)}-\frac{1}{2} e_{\nu}^{(\rho)} E^{\nu(\sigma)}+\frac{1}{2} e_{\nu}^{(\sigma)} E^{\nu(\rho)}=0 .
$$

Upon substitution of (15), contracted with the parameter of corresponding tensorial dimension and symmetries, $r_{(\gamma \sigma)}$, into (8), making simple rearrangements and integration by parts, we have

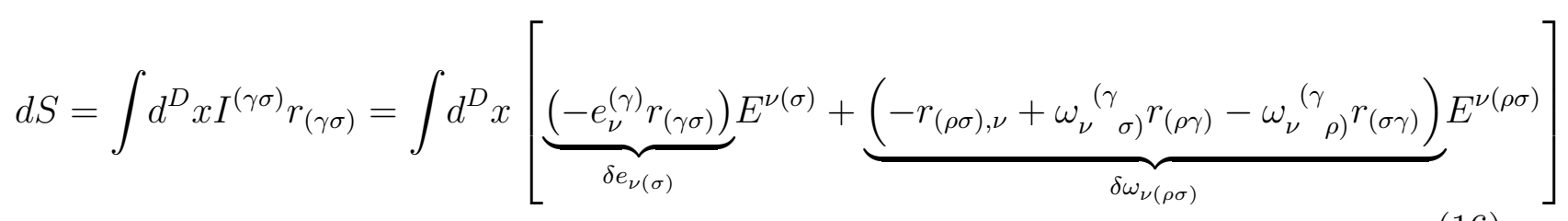


which gives the well-known rotational invariance for the first order N-bein gravity (the same invariance was derived using the Hamiltonian approach to N-bein gravity in [1, 2, 26])

$$
\begin{gathered}
\delta_{r} e_{\nu(\sigma)}=-e_{\nu}^{(\gamma)} r_{(\gamma \sigma)}, \\
\delta_{r} \omega_{\nu(\rho \sigma)}=-r_{(\rho \sigma), \nu}+\omega_{\nu}^{(\gamma}{ }_{\sigma)} r_{(\rho \gamma)}-\omega_{\nu}^{(\gamma}{ }_{\rho)} r_{(\sigma \gamma)} .
\end{gathered}
$$

Schwinger in [24] started from the transformations (17)-(18) (see page 1254, unnumbered equation in the middle of the second column) and from them obtained the identity (15). This is the standard way of "building" a DI by using a known invariance [21]. This actually underestimates the significance of the Lagrangian approach as DIs can be constructed from the Euler derivatives and consequently the invariance can be found from the DIs. Schwinger then used invariance of the fields $e_{\tau(\sigma)}$ and $\omega_{\tau(\rho \sigma)}$, which are both covariant vectors in external indices, under the infinitesimal coordinate transformations in the external space (diffeomorphism)

$$
\begin{gathered}
\delta_{d i f f} e_{\tau(\sigma)}=-e_{\tau(\sigma), \nu} \xi^{\nu}-e_{\nu(\sigma)} \xi_{, \tau}^{\nu}, \\
\delta_{d i f f} \omega_{\tau(\rho \sigma)}=-\omega_{\tau(\rho \sigma), \nu} \xi^{\nu}-\omega_{\nu(\rho \sigma)} \xi_{, \tau}^{\nu}
\end{gathered}
$$

and obtained the second DI by substitution of (19) and (20) into (8) and then converting it into the form of (7)

$$
I_{\nu}=-E^{\tau(\sigma)} e_{\tau(\sigma), \nu}+\left(E^{\tau(\sigma)} e_{\nu(\sigma)}\right)_{, \tau}-E^{\tau(\rho \sigma)} \omega_{\tau(\rho \sigma), \nu}+\left(E^{\tau(\rho \sigma)} \omega_{\nu(\rho \sigma)}\right)_{, \tau}=0
$$

(This is Schwinger's equation in our notation.) We note that $\delta_{\text {diff }}(.$.$) cannot be obtained$ from the first class constraints of the Hamiltonian formulation of the EC action [1, 26, 27] even though it is the transformation that leaves the action invariant.

Instead of (21), we will build the second DI in a way similar to the rotational one, starting from derivatives of the second ED (5). If such an identity exists, we have translational invariance in the tangent space. By performing explicit differentiation of (5) and using (A10) we obtain 
$\left.E_{, \tau}^{\tau(\rho)}=-e C^{\lambda(\sigma) \tau(\rho) \mu(\alpha) \nu(\beta)} e_{\lambda(\sigma), \tau}\left(\omega_{\nu(\alpha \beta), \mu}+\omega_{\mu(\alpha \gamma)} \omega_{\nu}{ }^{(\gamma}{ }_{\beta}\right)-e B^{\tau(\rho) \mu(\alpha) \nu(\beta)}\left(\omega_{\nu(\alpha \beta), \mu}+\omega_{\mu(\alpha \gamma)} \omega_{\nu}{ }^{(\gamma} \beta\right)\right)_{, \tau}$.

In contrast to the rotational DI (15), here we have terms quadratic in the derivatives $e_{\lambda(\sigma), \tau}$ and $\omega_{\nu(\alpha \beta), \mu}$. Terms with either $e_{\lambda(\sigma), \tau}$ or $\omega_{\nu(\alpha \beta), \mu}$ are in the EDs (5) and ([6) when contracted with $B$. This suggests an expansion of $C$ using the only one free (internal) index $(\rho)$ as in this case we have $B$ 's with all indices which appear in front of either $e_{\lambda(\sigma), \tau}$ or $\omega_{\nu(\alpha \beta), \mu}$. When compared with the last step in the derivation of the rotational DI (where we had a coefficient $A$ and it was necessary to contract $B$ with $\left.e_{\tau}^{(\sigma)}\right)$, the $\mathrm{ED}$ here is different: we have the coefficient $C$ which is the next "generation" of the $A B C$ functions and it has to be expanded in term of $B$ 's. Using (A8) we obtain

$$
\begin{gathered}
E_{, \tau}^{\tau(\rho)}=-e e^{\tau(\rho)} B^{\lambda(\sigma) \mu(\alpha) \nu(\beta)}\left(\omega_{\nu(\alpha \beta), \mu}+\omega_{\mu(\alpha \gamma)} \omega_{\nu \beta}^{(\gamma}\right) e_{\lambda(\sigma), \tau} \\
+e e^{\lambda(\rho)} B^{\mu(\sigma) \nu(\alpha) \tau(\beta)}\left(\omega_{\nu(\alpha \beta), \mu}+\omega_{\mu(\alpha \gamma)} \omega_{\nu \beta}^{(\gamma}\right) e_{\lambda(\sigma), \tau} \\
-e e^{\mu(\rho)} B^{\nu(\sigma) \tau(\alpha) \lambda(\beta)} e_{\lambda(\sigma), \tau}\left(\omega_{\nu(\alpha \beta), \mu}+\omega_{\mu(\alpha \gamma)} \omega_{\nu}^{(\gamma} \beta\right) \\
+e e^{\nu(\rho)} B^{\tau(\sigma) \lambda(\alpha) \mu(\beta)} e_{\lambda(\sigma), \tau}\left(\omega_{\nu(\alpha \beta), \mu}+\omega_{\mu(\alpha \gamma)} \omega_{\nu}^{(\gamma} \beta\right) \\
-e B^{\tau(\rho) \mu(\alpha) \nu(\beta)}\left(\omega_{\mu(\alpha \gamma)} \omega_{\nu}^{(\gamma} \beta\right)_{, \tau} \cdot
\end{gathered}
$$

(Here we used the identity (A13): $e B^{\tau(\rho) \mu(\alpha) \nu(\beta)} \omega_{\nu(\alpha \beta), \mu \tau}=0$.)

In the first two lines, we immediately have a full expression for the contracted ED (15) and in the third and fourth lines we have contributions which are equal to terms with derivatives in the contracted ED (6). As the result, we convert (23) into

$$
\begin{gathered}
E_{, \tau}^{\tau(\rho)}=e^{\tau(\rho)} E^{\lambda(\sigma)} e_{\lambda(\sigma), \tau}-e^{\lambda(\rho)} E^{\tau(\sigma)} e_{\lambda(\sigma), \tau} \\
+e^{\mu(\rho)} E^{\nu(\alpha \beta)}\left(\omega_{\nu(\alpha \beta), \mu}+\omega_{\mu(\alpha \gamma)} \omega_{\nu}{ }_{\nu}^{(\gamma}\right)-e^{\nu(\rho)} E^{\mu(\alpha \beta)}\left(\omega_{\nu(\alpha \beta), \mu}+\omega_{\mu(\alpha \gamma)} \omega_{\nu}^{(\gamma} \beta\right)+W^{(\rho)}
\end{gathered}
$$


where

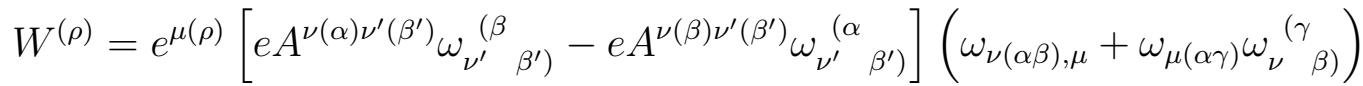

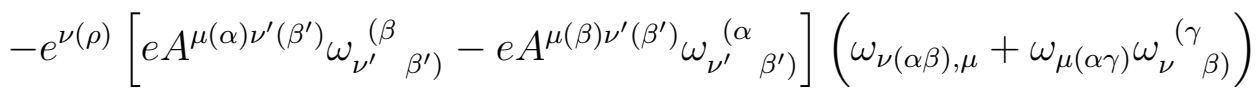

$$
\begin{aligned}
& \left.\left.-e B^{\tau(\rho) \mu(\alpha) \nu(\beta)}\left(\omega_{\mu(\alpha \gamma), \tau} \omega_{\nu}^{(\gamma} \beta\right)+\omega_{\mu(\alpha \gamma)} \omega_{\nu}^{(\gamma} \beta\right), \tau\right) .
\end{aligned}
$$

All terms with two derivatives have been expressed in terms of EDs from (5) and (6) and we are left only with terms linear in the derivatives and terms without derivatives which are of third order in the connections. We have to continue and try to express terms with derivatives again through EDs.

The derivatives presented in $W^{(\rho)}$ correspond to the ED (5) $E^{\lambda(\sigma)}$, contracted with $\omega_{\mu(\alpha \gamma)}$. Since $e^{\mu(\rho)} A^{\nu(\alpha) \nu^{\prime}\left(\beta^{\prime}\right)}$ has the dimension of $B$, we can try to expand $B$ to obtain such combinations. However, because $A$ (see (24)) has only two external indices that are contracted with indices of derivative of connections, it is not immediately in the form of (5). Performing an expansion of $B^{\tau(\rho) \mu(\alpha) \nu(\beta)}$ in a free internal index, (A7), we obtain

$$
e^{\mu(\rho)} A^{\nu(\alpha) \tau(\beta)}-e^{\nu(\rho)} A^{\mu(\alpha) \tau(\beta)}=-e^{\tau(\rho)} A^{\mu(\alpha) \nu(\beta)}-B^{\tau(\rho) \mu(\alpha) \nu(\beta)} .
$$

This is exactly the combination that we have in (24) and this allows us to convert (24) into a contraction of the expression that we used in our calculations of the rotational DI (11). In addition, after substitution of (25) the last term in (24) cancels. At this step, all derivatives have been eliminated and the rest of the terms, all of third order in the connections, can be easily analyzed. As in the rotational case, all of them cancel, and we have a new, translational, DI

$I^{(\rho)}=E_{, \nu}^{\nu(\rho)}-e^{\tau(\rho)} E^{\nu(\beta)} e_{\nu(\beta), \tau}+e^{\tau(\rho)} E^{\nu(\beta)} e_{\tau(\beta), \nu}-e^{\mu(\rho)} E^{\nu(\alpha \beta)} R_{\mu \nu(\alpha \beta)}+e^{\tau(\rho)} \omega_{\tau(\gamma \beta)} e_{\nu}^{(\gamma)} E^{\nu(\beta)}=0$.

Contracting this with the corresponding parameter $t_{(\rho)}$ (in a way similar to what was done for the rotational DI in (16)), we obtain 


$$
\int d^{D} x I^{(\rho)} t_{(\rho)}=0
$$

A simple rearrangement gives the transformations corresponding to this DI

$$
\begin{gathered}
\delta_{t} e_{\nu(\beta)}=-t_{(\beta), \nu}-e^{\tau(\rho)} e_{\nu(\beta), \tau} t_{(\rho)}+e^{\tau(\rho)} e_{\tau(\beta), \nu} t_{(\rho)}+e^{\tau(\rho)} \omega_{\tau(\gamma \beta)} e_{\nu}^{(\gamma)} t_{(\rho)}, \\
\delta_{t} \omega_{\nu(\alpha \beta)}=-e^{\mu(\rho)} R_{\mu \nu(\alpha \beta)} t_{(\rho)} .
\end{gathered}
$$

Note that the identity (26) can be rewritten in a slightly different form. If we add and subtract the term $\omega_{\nu(\beta}^{\rho)} E^{\nu(\beta)}$ and rewrite one of these terms as $e^{\tau(\rho)} e_{\tau}^{(\gamma)} \omega_{\nu(\beta \gamma)} E^{\nu(\beta)}$, then $I^{(\rho)}$ becomes

$$
I^{(\rho)}=E_{, \nu}^{\nu(\rho)}-\omega_{\nu(\beta}{ }^{\rho)} E^{\nu(\beta)}+e^{\tau(\rho)} T_{\nu \tau(\beta)} E^{\nu(\beta)}-e^{\mu(\rho)} E^{\nu(\alpha \beta)} R_{\mu \nu(\alpha \beta)}
$$

where

$$
T_{\nu \tau(\beta)} \equiv e_{\tau(\beta), \nu}-e_{\nu(\beta), \tau}+\omega_{\tau(\gamma \beta)} e_{\nu}^{(\gamma)}-\omega_{\nu(\gamma \beta)} e_{\tau}^{(\gamma)}
$$

is the torsion tensor (see, e.g. [28]), ${ }^{3}$ which is antisymmetric in external indices $\nu$ and $\tau$. With $I^{(\rho)}$ of (30), the transformation $\delta_{t} e_{\nu(\beta)}$ takes the form

$$
\delta_{t} e_{\nu(\beta)}=-t_{(\beta), \nu}-\omega_{\nu(\beta}{ }^{\rho)} t_{(\rho)}+e^{\tau(\rho)} T_{\nu \tau(\beta)} t_{(\rho)} .
$$

The first order formulation with two sets of independent variables leads to two sets of EDs, two the simplest, basic, DIs and two symmetries. Many other DIs can be constructed from these two basic identities and the corresponding symmetries of an action can be found, and one particular DI (out of many possible) gives the diffeomorphism

$$
I_{\nu}=e_{\nu(\alpha)} I^{(\alpha)}+\omega_{\nu(\gamma \beta)} I^{(\gamma \beta)},
$$

which coincides with (21).

\footnotetext{
${ }^{3}$ Note that $T_{\nu \tau(\beta)}$ is different from the torsion tensor $\Gamma_{[\alpha \beta]}^{\gamma}$ which appears in metric gravity when the affine connection $\Gamma_{\alpha \beta}^{\gamma}$ is assumed to be nonsymmetric: $\Gamma_{[\alpha \beta]}^{\gamma}=\frac{1}{2}\left(\Gamma_{\alpha \beta}^{\gamma}-\Gamma_{\beta \alpha}^{\gamma}\right)$ (see, e.g. [29]).
} 


\section{GROUP PROPERTIES OF THE TRANSFORMATIONS}

The algebraic properties of the transformations found in the previous Section can be analyzed by considering commutators of two successive transformations. (This is what Bergmann and Komar [31] used to discuss group properties of diffeomorphism invariance of the Einstein-Hilbert action where the basic DI indeed is the "world" vector, contrary to the basic DIs of the Einstein-Cartan action.)

We find the commutators between two transformations of fields and using this identify what algebra they obey. Let us start by calculating the commutator between two rotational transformations $\left(\delta_{r^{\prime \prime}} \delta_{r^{\prime}}-\delta_{r^{\prime}} \delta_{r^{\prime \prime}}\right) e_{\tau(\sigma)}$. Using (17) we obtain

$$
\left(\delta_{r^{\prime \prime}} \delta_{r^{\prime}}-\delta_{r^{\prime}} \delta_{r^{\prime \prime}}\right) e_{\tau(\sigma)}=-e_{\tau}^{(\lambda)} \bar{r}_{(\lambda \sigma)}=\delta_{\bar{r}} e_{\tau(\sigma)}
$$

where the new parameter is related to $r^{\prime}$ and $r^{\prime \prime}$ as

$$
\bar{r}_{(\lambda \sigma)} \equiv r_{(\lambda}^{\prime \gamma)} r_{(\gamma \sigma)}^{\prime \prime}-r_{(\lambda}^{\prime \prime \gamma)} r_{(\gamma \sigma)}^{\prime} .
$$

Similarly, using (18) we can find the commutator between two rotations for a connection

$$
\left(\delta_{r^{\prime \prime}} \delta_{r^{\prime}}-\delta_{r^{\prime}} \delta_{r^{\prime \prime}}\right) \omega_{\tau(\rho \sigma)}=-\bar{r}_{(\rho \sigma), \tau}+\omega_{\tau \sigma)}^{(\lambda} \bar{r}_{(\rho \lambda)}-\omega_{\tau}^{(\lambda)}{ }_{(\sigma \lambda)}=\delta_{\bar{r}} \omega_{\tau(\rho \sigma)}
$$

with the same parameter $\bar{r}_{(\lambda \sigma)}$ as in (34).

For the commutator between a rotation and a translation we obtain

$$
\begin{gathered}
\left(\delta_{r} \delta_{t}-\delta_{t} \delta_{r}\right) e_{\nu(\beta)}=-\left(r_{\beta)}^{(\rho} t_{(\rho)}\right)_{, \nu}+e^{\lambda(\gamma)} e_{\lambda(\beta), \nu}\left(r_{\gamma)}^{(\rho} t_{(\rho)}\right)-e^{\lambda(\gamma)} e_{\nu(\beta), \lambda}\left(r_{\gamma)}^{(\rho} t_{(\rho)}\right) \\
+e^{\tau(\sigma)} \omega_{\tau(\gamma \beta)} e_{\nu}^{(\gamma)}\left(r_{\sigma)}^{(\rho} t_{(\rho)}\right)=\delta_{\bar{t}} e_{\nu(\beta)}, \\
\left(\delta_{r} \delta_{t}-\delta_{t} \delta_{r}\right) \omega_{\tau(\rho \sigma)}=-e^{\mu(\beta)} R_{\mu \tau(\rho \sigma)}\left(r_{\beta)}^{(\gamma} t_{(\gamma)}\right)=\delta_{\bar{t}} \omega_{\tau(\rho \sigma)}
\end{gathered}
$$

with the new parameter

$$
\bar{t}_{(\beta)} \equiv r_{\beta)}^{(\rho} t_{(\rho)} .
$$

Calculation of the commutator between two translations is more involved. For $e_{\nu(\beta)}$ it gives 


$$
\begin{aligned}
\left(\delta_{t^{\prime \prime}} \delta_{t^{\prime}}-\delta_{t^{\prime}} \delta_{t^{\prime \prime}}\right) e_{\nu(\beta)} & =-e^{\lambda(\rho)} e^{\tau(\gamma)} R_{\lambda \tau(\alpha \beta)} t_{(\rho)}^{\prime} t_{(\gamma)}^{\prime \prime} e_{\nu}^{(\alpha)}-\left(e^{\lambda(\rho)} e^{\tau(\gamma)} T_{\lambda \tau(\beta)} t_{(\rho)}^{\prime} t_{(\gamma)}^{\prime \prime}\right)_{, \nu} \\
& -\omega_{\nu(\beta)}{ }^{\sigma)} e^{\lambda(\rho)} e^{\tau(\gamma)} T_{\lambda \tau(\sigma)} t_{(\rho)}^{\prime} t_{(\gamma)}^{\prime \prime}+e^{\mu(\sigma)} T_{\nu \mu(\beta)} e^{\lambda(\rho)} e^{\tau(\gamma)} T_{\lambda \tau(\sigma)} t_{(\rho)}^{\prime} t_{(\gamma)}^{\prime \prime} \\
& =\delta_{\tilde{r}} e_{\nu(\beta)}+\delta_{\tilde{t}} e_{\nu(\beta)}
\end{aligned}
$$

and for a connection $\omega_{\nu(\alpha \beta)}$ we obtain

$$
\begin{aligned}
\left(\delta_{t^{\prime \prime}} \delta_{t^{\prime}}-\delta_{t^{\prime}} \delta_{t^{\prime \prime}}\right) \omega_{\nu(\alpha \beta)} & =-\left(e^{\lambda(\rho)} e^{\tau(\gamma)} R_{\lambda \tau(\alpha \beta)} t_{(\rho)}^{\prime} t_{(\gamma)}^{\prime \prime}\right)_{, \nu} \\
& +e^{\lambda(\rho)} e^{\tau(\gamma)} t_{(\rho)}^{\prime} t_{(\gamma)}^{\prime \prime}\left(R_{\lambda \tau(\sigma \beta)} \omega_{\nu}^{(\sigma}{ }_{\alpha)}-R_{\lambda \tau(\sigma \alpha)} \omega_{\nu}{ }^{(\sigma}{ }_{\beta}\right) \\
& -e^{\lambda(\rho)} e^{\tau(\gamma)} t_{(\rho)}^{\prime} t_{(\gamma)}^{\prime \prime} T_{\lambda \tau(\sigma)} e^{\mu(\sigma)} R_{\mu \nu(\alpha \beta)} \\
& =-\tilde{r}_{(\alpha \beta), \nu}+\omega_{\nu}^{(\gamma} \tilde{r}_{(\alpha \gamma)}-\omega_{\nu}^{(\gamma}{ }_{\alpha} \tilde{r}_{(\beta \gamma)}-e^{\mu(\sigma)} R_{\mu \nu(\alpha \beta)} \tilde{t}_{(\sigma)} \\
& =\delta_{\tilde{r}} \omega_{\nu(\alpha \beta)}+\delta_{\tilde{t}} \omega_{\nu(\alpha \beta)}
\end{aligned}
$$

where the new parameters are

$$
\tilde{r}_{(\alpha \beta)} \equiv e^{\lambda(\rho)} e^{\tau(\gamma)} R_{\lambda \tau(\alpha \beta)} t_{(\rho)}^{\prime} t_{(\gamma)}^{\prime \prime}
$$

and

$$
\tilde{t}_{(\sigma)} \equiv e^{\lambda(\rho)} e^{\tau(\gamma)} T_{\lambda \tau(\sigma)} t_{(\rho)}^{\prime} t_{(\gamma)}^{\prime \prime} .
$$

Note that in the flat spacetime limit both $R_{\lambda \tau(\alpha \beta)}$ and $T_{\lambda \tau(\sigma)}$ are zero and in this limit the ordinary Poincaré symmetry is recovered. In addition, it is clear from (39) and (40) that the algebra of translations is not closed by itself (without rotation). In the second order formulation of the EC action only one ID, $I^{(\rho)}$, can be found from the variation of the Lagrangian with respect to $e_{\tau(\rho)}$ and the necessity of finding the second DI, $I^{(\alpha \beta)}$, is not obvious without having some a priori knowledge. Possibly, non-closure of the algebra of translations in the second order formulation provides a clue that we should seek another DI.

Commutators (33) / (35)), (36) / (37) and (39) / (40) form a closed algebra; (33) / (35) and (36) /(37) are part of the flat spacetime Poincaré algebra while the commutators (39) / (40) differs from Poincaré and similar to found in [3, 4]. Consequently, transformations that follow from the basic identities form the group with field dependent structure functions. 
These transformations are not the only ones that leave the EC action invariant, as many other DIs can be constructed that correspond to invariances of the action, and their group properties can be studied. For example, the identity (21) corresponds to diffeomorphism invariance of (19) and (20) and related to rotation and translation by (32). The commutator of two diffeomorphism transformations gives

$$
\left(\delta_{d i f f}^{\prime} \delta_{d i f f}^{\prime \prime}-\delta_{d i f f}^{\prime \prime} \delta_{d i f f}^{\prime}\right) e_{\nu(\lambda)}=-e_{\rho(\lambda)} \bar{\xi}_{, \nu}^{\rho}-e_{\nu(\lambda), \rho} \bar{\xi}^{\rho}=\delta_{d i f f} e_{\nu(\lambda)}
$$

with the new parameter

$$
\bar{\xi}^{\rho} \equiv \xi_{, \gamma}^{\prime \rho} \xi^{\prime \prime \gamma}-\xi_{, \gamma}^{\prime \prime} \rho \xi^{\prime \gamma}
$$

which is the same as found by Bergmann and Komar in [31].

We can also calculate the commutators among transformations that correspond to different symmetries, e.g. diffeomorphism and rotation

$$
\begin{aligned}
\left(\delta_{r} \delta_{\text {diff }}-\delta_{\text {diff }} \delta_{r}\right) e_{\nu(\lambda)} & =\delta_{r}\left(-e_{\rho(\lambda)} \xi_{, \nu}^{\rho}-e_{\nu(\lambda), \rho} \xi^{\rho}\right)-\delta_{\text {diff }}\left(-e_{\tau}^{(\gamma)} r_{(\gamma \sigma)}\right) \\
& =e_{\rho}^{(\gamma)} r_{(\gamma \lambda)} \xi_{, \nu}^{\rho}-\left(-e_{\tau}^{(\gamma)} r_{(\gamma \sigma)}\right)_{, \rho} \xi^{\rho}-\left(e_{\sigma}^{(\gamma)} \xi_{, \nu}^{\sigma}-e_{\nu, \sigma}^{(\gamma)} \xi^{\sigma}\right) r_{(\gamma \lambda)} \\
& =e_{\nu}^{(\gamma)} r_{(\gamma \lambda), \rho} \xi^{\rho}=-e_{\nu}^{(\gamma)} \hat{r}_{(\gamma \lambda)}=\delta_{\hat{r}} e_{\nu}^{(\gamma)}
\end{aligned}
$$

where

$$
\hat{r}_{(\gamma \lambda)} \equiv r_{(\gamma \lambda), \rho} \xi^{\rho},
$$

which shows the group property (it gives transformation under rotation again). Note that this is not equivalent with what is reported in [25, 30, 32] where it is claimed that the first class constraints that correspond to these transformations have zero PB. We found the only one paper where the PB between the generator of rotation ("Lorentz" transformation) and the generator of "three-dimensional general coordinate transformations" is proportional to the generator of rotation [33].

Finally we can consider a commutator of diffeomorphism and translational transformations. This leads to the following result

$$
\left(\delta_{t} \delta_{d i f f}-\delta_{\text {diff }} \delta_{t}\right) e_{\nu(\lambda)}=\left(t_{(\lambda), \rho} \xi^{\rho}\right)_{, \nu} .
$$


It is clear that these two transformations do not form a group. This is consistent with the fact that at the Lagrangian level diffeomorphism and translation are not independent as DI of diffeomorphism can be expressed as a linear combination of rotational and translational DIs (see (32)). At the Hamiltonian level, this probably is an indication that there is no canonical transformation between variables in which the corresponding Hamiltonian formulations of the same theory produce diffeomorphism and translation in the internal space as gauge symmetries, respectively.

By construction of the DIs, the EC action (11)-(2) is automatically invariant "off-shell" under the transformations (17)-(18) and (28)-(29). Direct demonstration of this is most easily done if the $A B C$ properties given in Appendix A are used. It is not difficult to check that the variation of the Lagrange density under rotation is $\delta_{r} L=0$ and under translation is $\delta_{t} L=-\left(e e^{\lambda(\rho)} R t_{(\rho)}\right)_{, \lambda}$ where $R=e^{\mu(\alpha)} e^{\nu(\beta)} R_{\mu \nu(\alpha \beta)}$.

In addition, because $L(e, \omega)$ is equivalent to the second order formulation $L(e)$, where $\omega_{\nu(\alpha \beta)}$ is expressed in terms of $e_{\nu(\beta)}$ by using its equation of motion and is not an independent field, it is unnecessary to recalculate the DI to find transformations for $L(e)$. The solution for connections in terms of N-beins is known and can be presented in terms of $A^{\gamma(\tau) \mu(\varepsilon)}$ (see [1])

$$
\omega_{\nu}^{(\tau \lambda)}=\frac{1}{2} e_{\nu(\varepsilon)}\left(A^{\gamma(\tau) \mu(\varepsilon)} e_{\gamma, \mu}^{(\lambda)}+A^{\gamma(\varepsilon) \mu(\lambda)} e_{\gamma, \mu}^{(\tau)}-A^{\gamma(\lambda) \mu(\tau)} e_{\gamma, \mu}^{(\varepsilon)}\right)
$$

Upon substitution of (48) into (31) we obtain

$$
\delta_{t} e_{\nu(\beta)}=-t_{(\beta), \nu}-\omega_{\nu(\beta}^{\rho)} t_{(\rho)} .
$$

The transformation of $e_{\nu(\beta)}$ under a rotation does not change when passing to the second order formulation because (17) does not depend on the connection. The second transformation, (29), for the second order formulation can be derived from (48) and (49) if we express $R_{\mu \nu(\alpha \beta)}$ in terms of $e_{\nu(\beta)}$ and its derivatives. All term proportional to $T_{\nu \tau(\beta)}$ disappear in (49) if we move to the second order formalism.

Note that the connection $\omega_{\nu(\beta}^{\rho)}$ in (49) is a function of $e_{\nu(\beta)}$ given in (48) and is not an independent variable, if we consider the second order formalism. Ironically, exactly this equation, (49), has been used for decades as the main argument that the EC action in not invariant under a translation in dimensions higher than three. In the literature, equation 
(49) is often accompanied by $\delta_{t} \omega_{\nu(\alpha \beta)}=0$ rather than (29) [34] (though in three dimensions it is indeed true that $\left.\delta_{t} \omega_{\nu(\alpha \beta)}=0\right)$.

The transformations (17), (18) and (31), (29) are not new and have been presented, e.g. in [3, 22]. However, the question whether these transformations represent the gauge symmetry which follows from the Hamiltonian analysis was not discussed.

We consider basic IDs, (15) and (26), and one well known but not basic, (21), which gives diffeomorphism transformation, but many other DIs and symmetries can be found and group properties of the corresponding transformations can be studied. All of them, as in the simple example above (see (47)), cannot be combined into a group but different groups can be formed (e.g. diffeomorphism and rotation form a group as do translation and rotation), but not every group follows from the first class constraints. The Hamiltonian analysis leads uniquely to the gauge invariance (what follows from the structure of first class constraints) without any ambiguity. Preliminary results of the Hamiltonian formulation of the EC action [1, 2] show that even from first simple steps of the Dirac procedure, from the tensorial dimension of primary first class constraints, the translation and rotation in the tangent space follow unambiguously as a gauge symmetry. In conclusion we argue that the Lagrangian methods also allow us to single out a unique gauge invariance of any theory, the same invariance as follows from the Hamiltonian analysis.

\section{CONCLUSION}

What invariances are true gauge invariances of the N-bein formulation of gravity? "What is a gauge symmetry and what is not?" [23]. The rotational and translational transformations, (17), (18) and (31), (29), both have internal parameters and if a rotational invariance is a gauge invariance, then should translational invariance. In the Hamiltonian analysis of the first order formulation of N-bein gravity, exactly these two parameters emerge, both with only internal indices [1, 2, 26]. This result which contradicts conventional wisdom motivated us to perform the short investigation reported here, in which we examine what invariances appear in the Lagrangian approach. In the Hamiltonian formulation, where elimination of the second class constraints is needed [17], restoration of the manifestly covariant form of the transformations is difficult. If one is not interested in finding the constraints and their

algebra, the manifestly covariant Lagrangian method is simpler. However, there is an appar- 
ent "deficiency" in the Lagrangian method: we presented here a few differential identities (see (15), (21), (26) ), but linear combinations of these identities will lead to additional invariances of an original action. These cannot all be gauge symmetries generated by the first class constraints. This is clear, as in the Hamiltonian formulation each gauge symmetry originates from primary first class constraints and, if all symmetries are to be accommodated, more first class constraints than variables would be needed. This would be inconsistent as it leads to a negative number of degrees of freedom which is an unacceptable result. Similarly, both translational and diffeomorphism invariances cannot be simultaneously gauge symmetries arising from the first class constraints in any Hamiltonian formulation of N-bein gravity, though both are symmetries of the original action. We have also shown that at the Lagrangian level they do not form a group (47). Indeed, it follows, for example, from (32) that three invariances, rotation, translation and diffeomorphism, are not independent. Only linearly independent identities contribute to independent symmetries of an action [21].

In the Hamiltonian formulation of the first order EC gravity when using the original variables, $e_{\tau(\rho)}$ and $\omega_{\nu(\alpha \beta)}$, diffeomorphism cannot be derived from the first class constraints as a gauge symmetry (as we have shown in [1, 2, 26]). In addition, diffeomorphism has never been obtained for N-bein gravity in the Hamiltonian formulation without some unjustified assumptions, such as using a non-canonical change of variables, solving a first class constraint, or fixing a "gauge" before the gauge symmetry was derived. In the Hamiltonian formulation, both translational and diffeomorphism symmetries cannot simultaneously be present as gauge symmetries and the only possibility of reconciling these two symmetries as being gauge symmetries would be to find a canonical transformation between the original variables, $e_{\gamma(\rho)}$ and $\omega_{\nu(\alpha \beta)}$, and some others in terms of which we have diffeomorphism invariance. ${ }^{4}$ However, this is a faint possibility that such a transformation exists and it is more likely that canonical transformations do not change a gauge symmetry arising from the first class constraints [36] (see also Section 5 of [27]).

What we can call a gauge symmetry is not to be determined by our choice and all arguments based on "convenience" or "custom" or referred to as being "physical", "geometrical" are not acceptable. A mathematical criterion has to be developed to answer this question.

\footnotetext{
${ }^{4}$ Of course, having solely spatial diffeomorphism for a covariant theory is not acceptable. From the Hamiltonian point of view it guarantees that one has performed a non-canonical change of variables [27, 35].
} 
It is dangerous and purely speculative to draw any conclusion about a gauge symmetry without justifying it with the well-defined mathematical procedure. In any case, the gauge symmetries of a system (i.e. those invariances which follow from the first class constraints present) must be found by following the well prescribed procedure.

In the Lagrangian approach, there is nothing special in the question "diffeomorphism versus translational invariance", as many differential identities can be built starting from basic identities and their combinations. We can construct even different forms of translational invariance with the same, internal, index by using, in addition to the naturally occurring $I^{(\alpha)}$ (26) (let us call this the "zero DI", $I_{0}^{(\alpha)}$ ), other differential identities; for example, from $I_{\mu}$ (21) and $I^{(\alpha \beta)}$ (15) we can construct $I_{1}^{(\alpha)}=e^{\mu(\alpha)} \omega_{\mu(\gamma \beta)} I^{(\gamma \beta)}, I_{2}^{(\alpha)}=e^{\mu(\alpha)} I_{\mu}$, etc., and obtain from them the corresponding transformations. Moreover, for DIs of the same tensorial dimension we can consider linear combinations of them with real coefficients $I^{(\alpha)}=\sum_{i=0}^{n} a_{i} I_{i}^{(\alpha)}$ which lead to any number of translational symmetries. (Note that not all $I_{i}^{(\alpha)}$ are independent; for example, $I_{2}^{(\alpha)}=I_{0}^{(\alpha)}+I_{1}^{(\alpha)}$.) However, all these symmetries cannot be generated simultaneously by the first class constraints in the Hamiltonian formulation. Can all of them be gauge symmetries (but not simultaneously)? Which of these "translations" form a group and exhibit group properties with, for example, diffeomorphism and rotation? Translational invariance and diffeomorphism together do not form a group (see (47)) but the commutator of diffeomorphism and rotation does so (45)), and thus from the Lagrangian point of view they form a group [21]. This result contradicts to the "canonical" formulation of the EC action where it is claimed that the PB among constraints responsible for diffeomorphism and rotation are zero [25, 30, 32] which is an additional indication that these results should be checked for correctness.

Finding translational invariance in N-bein gravity in any dimension is a very simple task in the Lagrangian approach. ${ }^{5}$ The derivation of the translational invariance of N-bein gravity in any dimension leads to a much more general question: which symmetries of an action can

\footnotetext{
${ }^{5}$ The exception is the three dimensional case where totally antisymmetric $C^{\tau(\rho) \lambda(\sigma) \mu(\alpha) \nu(\beta)}$ in (22) (that has four indices internal or external) is manifestly zero (see (A6) or (A8)) and so it is necessary in the Lagrangian approach to treat the three dimensional case separately in order to find its translational invariance. This is also consistent with the Hamiltonian formulation where in $D=3$ some peculiarities appear that are not present in higher dimensions [2]. For peculiarities of sypersymmetric extension in $D=3$ see [37, 38].
} 
be called gauge symmetries? The Hamiltonian approach allows us to address this question and uniquely derive the gauge invariance for a particular formulation of any theory. This is important, as in methods of quantization, such as in path integral quantization using the Faddeev-Popov technique, it is necessary to fix a gauge and one must know which symmetry (in the case of N-bein gravity) has to be fixed (internal translation, and which one $\left(I_{0}^{(\alpha)}, I_{1}^{(\alpha)}, I_{2}^{(\alpha)}, \ldots\right)$, or restrictions on possible coordinate transformations have to be imposed). Some authors (see, e.g. [39]) have attempted to quantize vierbein gravity using a gauge fixing which breaks rotational and diffeomorphism invariances, without considering translational invariance. However, any conclusion about the quantum behaviour of gravity should be made only after we understand which gauge has to be fixed.

If the transformations (17), (18) and (31), (29) are to be considered as the gauge transformations, then the Hamiltonian formulation of the EC action has to have first class constraints which lead to a gauge generator that generates these transformations without any field dependent redefinition of gauge parameters.

It is possible that the Lagrangian approach can be used to single out which symmetry is a gauge symmetry, that can be derived from the structure of first class constraints of the Hamiltonian formulation of the same theory. This conjecture is based on the following observation [40]: in field theories (e.g. Maxwell, Yang-Mills, Einstein-Hilbert in its first or second order formulations) the basic differential identities formed from the derivatives of EDs lead to the invariance, exactly the same that follows from the first class constraints in the Hamiltonian formulation of a theory. In the EC action, the basic differential identities are (15) and (26) and these lead to translation and rotation in the tangent space. This is a pure Lagrangian result; we expect the same in the Hamiltonian formulation. The equivalence of Hamiltonian and Lagrangian methods leads to this conclusion and this equivalence also shows itself at some intermediate steps; for example, the commutators of transformations following from basic DIs and PBs among first class constraints responsible for such transformations are in direct correspondence for known gauge theories [40]. For the EC action the commutator of two rotations gives a rotation, (33)/(35), as when one computes the PB between two rotational constraints in the Hamiltonian formulation [1, 2]; the commutator of rotation and translation is proportional to translation only, (36)/(37), as we observe in the Hamiltonian formulation of the EC action [1, 2]. The commutator of two translations is proportional to a translation and a rotation; this must also be found for the PB of the 
corresponding constraints in the Hamiltonian formulation. The complete Hamiltonian formulation of the EC action is currently being investigated and the results will be reported elsewhere. In addition, there is a correspondence that has to be studied in which the commutators of transformations following from basic DIs can provide a clue about the PB algebra of the first class constraints. If in the commutator of two consecutive transformations the new parameter is expressed through old parameters without involving fields (as in (34) and (38)), then the PB between the corresponding constraints does not have field dependent structure functions. If there are no derivatives of parameters in the expression for the new parameter, then the corresponding PB algebra is local (i.e. has no derivatives of delta functions). For example, for the second order the Einstein-Hilbert action, the Lagrangian approach leads to the known result (44) (see [31]), in which the new parameter involves derivatives of old parameters and the corresponding PB algebra of constraints is non-local in this case. These general properties of the Lagrangian and Hamiltonian formulations of field theories should be applicable to the EC action and this is exactly what we have already found by considering the Lagrangian method in this paper and the preliminary results following from the Hamiltonian formulation in [1, 2, 26]. This provides an answer to Matschull's question posted for the EC theory: "what is a gauge symmetry and what is not". The gauge, basic, invariances of the EC action which follow from its first class constraints are translation and rotation in the tangent space, but not diffeomorphism. We found that the differential identities also play important role in analyzing consistency of coupling of gravity with other fields. The results will be reported elsewhere.

\section{Acknowledgements}

We would like to thank A.M. Frolov, D.G.C. McKeon, and J. Nowak for helpful discussion and reading the manuscript.

\section{Appendix A: $A B C$ properties}

Here we collect properties of the $A B C$ functions that were introduced in considering the Hamiltonian formulation of N-bein gravity [1, 2]. They turn out to also be very useful in the Lagrangian approach.

These functions are generated by consecutive variation of the N-bein density 


$$
\begin{gathered}
\frac{\delta}{\delta e_{\nu(\beta)}}\left(e e^{\mu(\alpha)}\right)=e\left(e^{\mu(\alpha)} e^{\nu(\beta)}-e^{\mu(\beta)} e^{\nu(\alpha)}\right)=e A^{\mu(\alpha) \nu(\beta)}, \\
\frac{\delta}{\delta e_{\lambda(\gamma)}}\left(e A^{\mu(\alpha) \nu(\beta)}\right)=e B^{\lambda(\gamma) \mu(\alpha) \nu(\beta)}, \\
\frac{\delta}{\delta e_{\tau(\sigma)}}\left(e B^{\lambda(\gamma) \mu(\alpha) \nu(\beta)}\right)=e C^{\tau(\sigma) \lambda(\gamma) \mu(\alpha) \nu(\beta)}, \quad \ldots
\end{gathered}
$$

The first important property of these density functions is their total antisymmetry: interchange of two indices of the same nature (internal or external), e.g.

$$
A^{\nu(\beta) \mu(\alpha)}=-A^{\nu(\alpha) \mu(\beta)}=-A^{\mu(\beta) \nu(\alpha)}
$$

with the same being valid for $B, C$, etc. In calculations presented here, nothing is needed beyond $C$.

The second important property is their expansion using an external index

$$
B^{\tau(\rho) \mu(\alpha) \nu(\beta)}=e^{\tau(\rho)} A^{\mu(\alpha) \nu(\beta)}+e^{\tau(\alpha)} A^{\mu(\beta) \nu(\rho)}+e^{\tau(\beta)} A^{\mu(\rho) \nu(\alpha)},
$$

$$
C^{\tau(\rho) \lambda(\sigma) \mu(\alpha) \nu(\beta)}=e^{\tau(\rho)} B^{\lambda(\sigma) \mu(\alpha) \nu(\beta)}-e^{\tau(\sigma)} B^{\lambda(\alpha) \mu(\beta) \nu(\rho)}+e^{\tau(\alpha)} B^{\lambda(\beta) \mu(\rho) \nu(\sigma)}-e^{\tau(\beta)} B^{\lambda(\rho) \mu(\sigma) \nu(\alpha)}
$$

or an internal index

$$
\begin{gathered}
B^{\tau(\rho) \mu(\alpha) \nu(\beta)}=e^{\tau(\rho)} A^{\mu(\alpha) \nu(\beta)}+e^{\mu(\rho)} A^{\nu(\alpha) \tau(\beta)}+e^{\nu(\rho)} A^{\tau(\alpha) \mu(\beta)}, \\
C^{\tau(\rho) \lambda(\sigma) \mu(\alpha) \nu(\beta)}=e^{\tau(\rho)} B^{\lambda(\sigma) \mu(\alpha) \nu(\beta)}-e^{\lambda(\rho)} B^{\mu(\sigma) \nu(\alpha) \tau(\beta)}+e^{\mu(\rho)} B^{\nu(\sigma) \tau(\alpha) \lambda(\beta)}-e^{\nu(\rho)} B^{\tau(\sigma) \lambda(\alpha) \mu(\beta)} .
\end{gathered}
$$

The third property involves their derivatives

$$
\begin{gathered}
\left(e A^{\nu(\beta) \mu(\alpha)}\right),_{\sigma}=\frac{\delta}{\delta e_{\lambda(\gamma)}}\left(e A^{\nu(\beta) \mu(\alpha)}\right) e_{\lambda(\gamma), \sigma}=e B^{\lambda(\gamma) \nu(\beta) \mu(\alpha)} e_{\lambda(\gamma), \sigma} \\
\left(e B^{\tau(\rho) \nu(\beta) \mu(\alpha)}\right),_{\sigma}=\frac{\delta}{\delta e_{\lambda(\gamma)}}\left(e B^{\tau(\rho) \nu(\beta) \mu(\alpha)}\right) e_{\lambda(\gamma), \sigma}=e C^{\tau(\rho) \lambda(\gamma) \nu(\beta) \mu(\alpha)} e_{\tau(\rho), \sigma}
\end{gathered}
$$


We also use the contraction of $B(\underline{\mathrm{A} 5})$ with a covariant $e_{\tau(\lambda)}$ :

$$
e_{\tau(\lambda)} B^{\tau(\rho) \mu(\alpha) \nu(\beta)}=\delta_{(\lambda)}^{(\rho)} A^{\mu(\alpha) \nu(\beta)}+\delta_{(\lambda)}^{(\alpha)} A^{\mu(\beta) \nu(\rho)}+\delta_{(\lambda)}^{(\beta)} A^{\mu(\rho) \nu(\alpha)} .
$$

Upon using the antisymmetry of $B$ both in the external and internal indices and the antisymmetry of $\omega$ in its internal indices leads to

$$
B^{\tau(\rho) \mu(\alpha) \nu(\beta)} \omega_{\mu(\alpha \gamma)} \omega_{\nu}^{(\gamma}{ }^{(\gamma} \omega_{\tau}^{(\sigma)}=0
$$

and

$$
e B^{\tau(\rho) \mu(\alpha) \nu(\beta)} \omega_{\nu(\alpha \beta), \mu \tau}=0
$$

A similar property for $C$ is

$$
C^{\lambda(\gamma) \nu(\alpha) \mu(\rho) \tau(\sigma)} e_{\lambda(\gamma), \tau} e_{\nu(\alpha), \mu}=0
$$

The above properties considerably simplify calculations. The list of $A B C$ properties can be extended, but for our purpose the above relations are adequate.

[1] N. Kiriushcheva and S.V. Kuzmin, arXiv:0907.1553 [gr-qc].

[2] A.M. Frolov, N. Kiriushcheva and S.V. Kuzmin, arXiv:0902.0856 [gr-qc] (to appear in Grav. \& Cosm.).

[3] F.W. Hehl, P. von der Heyde, G.D. Kerlick, J.M. Nester, Rev. Mod. Phys. 48 (1976) 393-416.

[4] A. Trautman, Ann. N.Y. Acad. Sci. 262 (1975) 241-245.

[5] P. Breitenlohner, Phys .Lett. B 67 (1977) 49-51.

[6] C. Teitelboim, Phys. Rev. Lett. 38 (1977) 1106-1110.

[7] M. Blagojević, Gravitation and Gauge symmetries, Institute of Physics Publishing, Bristol and Philadelphia, 2002.

[8] M. Bañados and M. Contreras, Class. Quantum Grav. 15 (1998) 1527-1534.

[9] E. Witten, Nucl. Phys. B 311 (1988) 46-78.

[10] D. Grensing and G. Grensing, Phys. Pev. D 28 (1983) 286-296.

[11] S. Carlip, Phys. Rev. D 42 (1990) 2647-2654. 
[12] S.A. Ali, C. Cafaro, S. Capozziello, Ch. Corda, Int. J. Theor. Phys. 48 (2009) 3426-3448.

[13] T.W.B. Kibble, Journal of Math. Phys. 2 (1961) 212-221.

[14] I.A. Nicolić, Class. Quantum Grav. 12 (1995) 3103-3114.

[15] S. Carlip, Quantum Gravity in 2+1 Dimensions, Cambridge University Press, Cambridge, 1998.

[16] J.M. Charap, M. Henneaux and J.E. Nelson, Class. Quantum Grav. 5 (1988) 1405-1414.

[17] P.A.M. Dirac, Lectures on Quantum Mechanics, Belfer Graduate School of Sciences, Yeshiva University, New York, 1964.

[18] L. Castellani, Ann. Phys. 143 (1982) 357-371.

[19] N. Kiriushcheva and S.V. Kuzmin, Ann. Phys. 321 (2006) 958-986.

[20] S. Samanta, Int. J. Theor. Phys. 48 (2009), 1436-1448, arXiv:0708.3300 [hep-th].

[21] E. Noether (M.A. Tavel's English translation), arXiv:physics/0503066.

[22] M. Leclerc, Int. J. Mod. Phys. D 16 (2007) 655-680.

[23] H.-J. Matschull, Class. Quantum Grav. 16 (1999) 2599-2609.

[24] J. Schwinger, Phys. Rev. 130 (1963) 1253-1258.

[25] L. Castellani, P. van Nieuwenhuizen, M. Pilati, Phys. Rev. D 26 (1982) 352-367.

[26] N. Kiriushcheva and S.V. Kuzmin, arXiv:0912.5490 [gr-qc].

[27] N. Kiriushcheva and S.V. Kuzmin, arXiv:0912.3396 [gr-qc].

[28] S. Deser and B. Zumino, Phys. Lett. B 62 (1976) 335-337.

[29] M. Carmeli, Classical Fields, General Relativity and Gauge Theory, World Scoentific, New Jersey, 2001.

[30] M. Henneaux, Phys. Rev. D 27 (1983) 986-989.

[31] P.G. Bergmann and A. Komar, Int. J. Theor. Phys. 5 (1972) 15-28.

[32] J.W. Maluf, Class. Quantum Grav. 8 (1991) 287-295.

[33] R. Di Stefano and R.T. Rauch, Phys. Rev. D 26 (1982) 1242-1253.

[34] G. Grignani and G. Nardelli, Phys. Rev. D 45 (1992) 2719-2731.

[35] N. Kiriushcheva and S.V. Kuzmin, arXiv:0809.0097 [gr-qc].

[36] A.M. Frolov, N. Kiriushcheva and S.V. Kuzmin, arXiv:0809.1198 [gr-qc].

[37] H. Nicolai, Nucl. Phys. B 353 (1991) 493-518.

[38] H.-J. Matschull and H. Nicolai, Nucl. Phys. B 411 (1994) 609-646.

[39] S. Deser, P. van Nieuwenhuizen, Phys. Rev. D 10 (1974) 411-420. 
[40] N. Kiriushcheva and S.V. Kuzmin, in preparation. 